\title{
Orthodontic Treatment of Severe Crowding Malocclusion with Extraction of premolars.
}

Rizvi $\mathrm{HM}^{1}$ BDS, FCPS, Sikder MA ${ }^{2}$ BDS, PhD and Hossain $\mathrm{MZ}^{3}$ BDS, PhD

\begin{abstract}
:
Space deficiency in a crowded dentition is a main indication for premolar extraction therapy. Class I malocclusions with severe crowding in non growing patients can be treated by upper lower first premolar extraction using an edgewise technique with multi loop edgewise arch wire (MEAW). The following case report describes a patient with severe tooth crowding, where the result of the treatment was acceptable and relatively stable. It was concluded that the elimination of tooth tissue discrepancy by the extraction of first premolars provides a good means in the treatment of crowding malocclusions.
\end{abstract}

Key words: Crowding, Molar extraction, MEAW. (Bangladesh Journal of Orthodontics and Dentofacial Orthopedics, Vol. 2, No. 2, April 2012, p 30-33).

\section{INTRODUCTION}

The dento-alveolar incongruence with crowding is a frequently seen malocclusion among the population, with a various etiology. ${ }^{1}$ The morpho-functional and masticator implications of such cases lead to a poor prognosis. Therefore it requires a correct treatment at the moment of diagnosis. The present work is motivated by the following observations:

1. Dental crowding has great negative influences on the dentomaxillary system. Besides the esthetic problem, which is the main factor motivating the patient to ask for dental treatment, there are other functional consequences, which can constitute favorizing factors for the onset and evolution of periodontal disease. $^{2}$

2. The effects of anterior or posterior dental crowding on the marginal periodontium include ${ }^{3}$ :

- Plaque retention and the difficulty in maintaining a good oral hygiene;

- Pathologic changes in gingival contour, Interdental bone and facial-lingual alveolar margin;

- Periodontal therapy (scaling, root planning, regenerative surgery) is more difficult to be performed in areas of crowding.
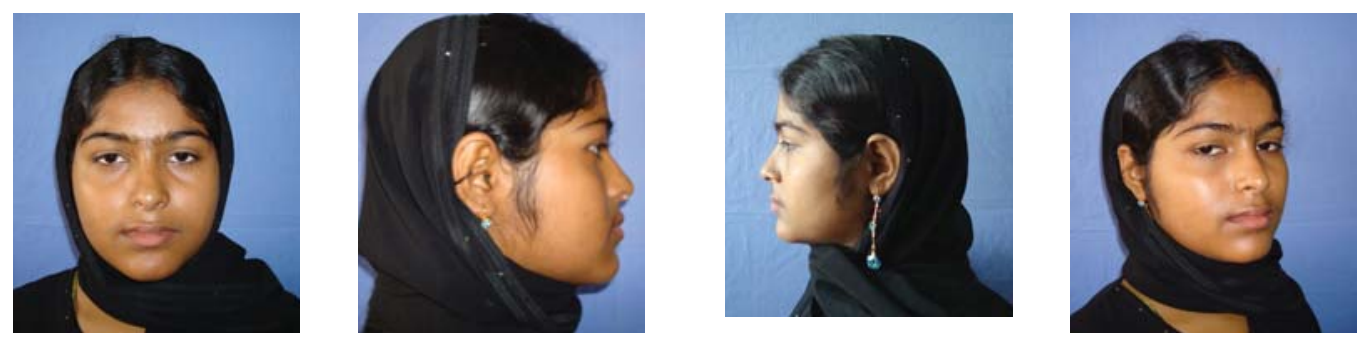

Fig. 1 before treatment extra oral photographs

3. Orthodontic adjustment of crowding has many esthetic and functional benefits and also will facilitate the maintenance of good oral hygiene, which is the key point to be considered for patients with severe crowding. ${ }^{4}$

\section{CASE DESCRIPTION}

The patient was a 18 years old female (Fig. 1). This case was presented by her parents because of the desire to correct the severe upper and lower tooth crowding (Fig. 2). Her face appeared to be symmetrical with a slightly convex profile. The patient exhibited a Class I molar relationship on both right and left sides of the dentition. Additionally, there were buccally placed maxillary left canine both the maxillary canines were also distally inclined and rotated. Presence of extra lateral incisors in the upper jaw. There was severe tooth imbrication of the anterior segment.

A model analysis revealed a severe tooth to denture base discrepancy, particularly in the upper jaw $(-18 \mathrm{~mm})$ and also in the lower jaw (-8) mm. A panoramic X-ray (Fig. 3) showed that four third molars were present and there were presences of supplemental lateral incisors in the upper arch.

Cephalometric evaluation indicated upper and lower jaw relation within the normal range however upper incisors were severely proclined and lower lip protrusive. (Fig. 4; Table 1)

30

$1{ }^{1}$ Dental Surgeon, Dept of Orthodontics, Dhaka Dental College and Hospital. ${ }^{2}$ Associate Professor and Head, Department of Orthodontics, Bangladesh Dental College and Hospital. ${ }^{3}$ Professor and head, Dept of Orthodontics, Dhaka Dental College and Hospital. 

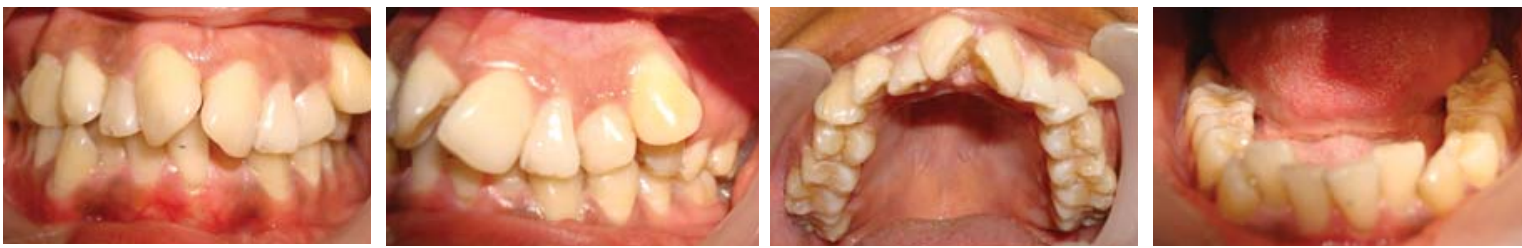

Fig. 2 Pre treatment intra oral photographs

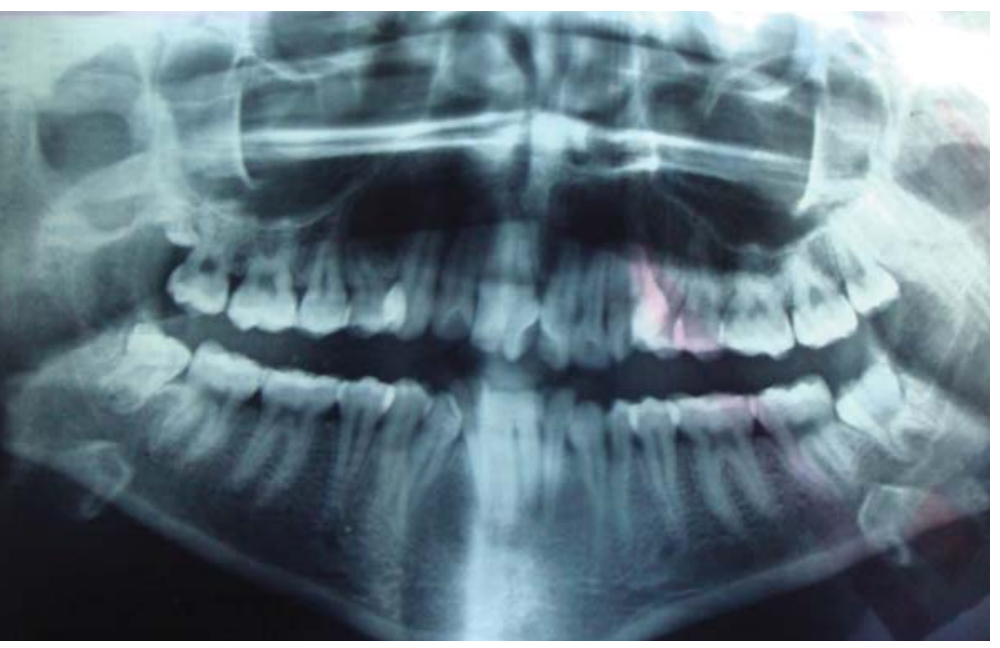

Fig. 3 Pre-treatment OPG

Table 1

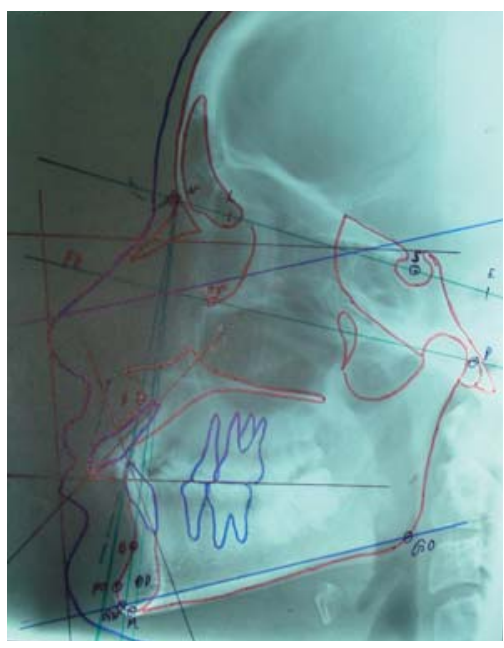

Fig. 4 Pre-treatment cephalometric tracing Lower lip appears to touch E line hence is protrusive
Parameter

Accepted standards for the Caucasian population according to Steiner analysis (age group $21-27$

years)

$\begin{array}{lcc}\text { SNA (angle) } & 82.01^{\circ} & 84^{\circ} \\ \text { SNB (angle) } & 79.97^{\circ} & 81^{\circ} \\ \text { ANB (angle) } & 2.04^{\circ} & 3^{\circ} \\ \text { SND (angle) } & 76^{\circ} & 79.5^{\circ} \\ \text { Upper 1 to NA (mm) } & 4 \mathrm{~mm} & 11.0 \mathrm{~mm} \\ \text { Upper 1 to NA (angle) } & 22.0^{\circ} & 33^{\circ} \\ \text { Lower 1 to NB (mm) } & 4 \mathrm{~mm} & 5.0 \mathrm{~mm} \\ \text { Lower 1 to NB (angle) } & 25.0^{\circ} & 28^{\circ} \\ \text { Inter incisal angle } & 127.0^{\circ} & 117^{\circ} \\ \text { OP to SN (angle) } & 14.0^{\circ} & 14^{\circ} \\ \text { Go Gn to SN (angle) } & 31.73^{\circ} & 29^{\circ} \\ \text { SE (mm) } & 22 & 21.5 \mathrm{~mm} \\ \text { SL(mm) } & 51 & 54.5 \mathrm{~mm}\end{array}$

Bangladesh Journal of Orthodontics and Dentofacial Orthopedics (BJO \& DFO) 


\section{DIAGNOSIS}

This case was diagnosed as having upper and lower crowding due to tooth to denture base discrepancy. Upper incisors rotated and tipped forward. Presence of supplemental lateral incisors in the upper arch. Maxillary left canine buccally placed; both the maxillary canines were also distally inclined and rotated.

\section{TREATMENT OBJECTIVES}

The treatment plan was to correct malaligned dentitions by extraction of all 1st premolars followed by alignment up righting of buccally placed, distally inclined and rotated canines. Contraction of the arches to correct overjet, overbite and improve esthetics. Interdigitation and arch coordination.

\section{PROGRESSION OF ORTHODONTIC TREATMENT}

After extraction of upper and lower 1st premolars, standard edgewise brackets were placed on all teeth. Segmental mechanics was used to retract and upright the maxillary canines (Fig. 5). Treatment was initiated with 0.014-inch Multi loop archwire to level all the teeth, and coil springs were placed to create the space for the blocked out teeth(Fig. 6). At six months of treatment, extraction of supplemental lateral incisors done to reduce overjet. $0.17 \times 0.025$ inch edgewise archwires was placed on the upper and lower dentitions in order to upright the posterior teeth and to provide the space for anterior teeth alignment.

$0.016 \times 0.022$ inch contraction edgewise archwires with reverse curve were used to contract the arches. A moderate tip hack bend was incorporated into them. 18 months of therapy provided a good interdigitarion in Class I molar relation and acceptable occlusal guidance, the edgewise appliance was removed and removable retention appliances were inserted. Constant yearly recall and documentation of the case showed maintenance of all supporting structures and occlusion.
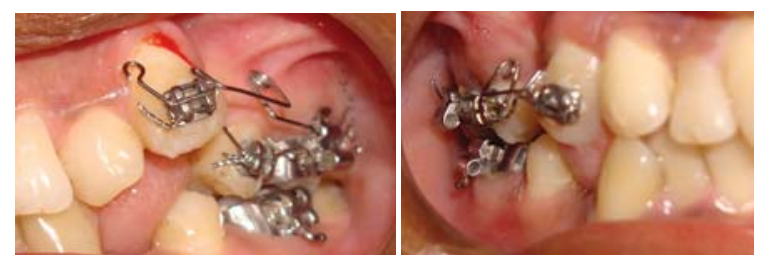

Fig. 5 retraction of upper canines

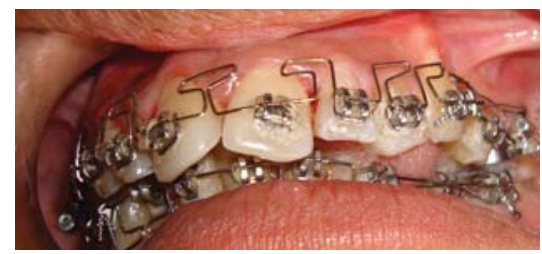

Fig. 6 leveling with 0.014-inch Multi loop archwire

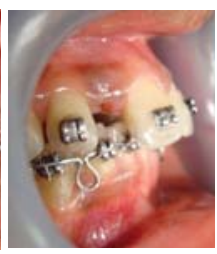

Fig. 7 extraction of supplemental lateral incisors

\section{THE TREATMENT RESULT}

Showed an improvement in the occlusion (Fig. 8). The crowding was eliminated and the inclination of the guiding plane and sequential functional guidance with canine dominance was achieved.(fig 9) Post treatment cephalogram tracings of before and after active orthodontic treatment indicated that the mandible grew in the downward and forward direction with a slight opening of the mandibular plane (Fig.10, table2). The upper anterior teeth were positioned backward, and the lower incisors compensated by inclining slightly lingually reducing the lower lip prominence. The post treatment panoramic radiograph showed acceptable root parallelism, although the canines could have been better with the roots more mesial (fig 11).

Fig. 8 Pre-post treatment intra oral photographs
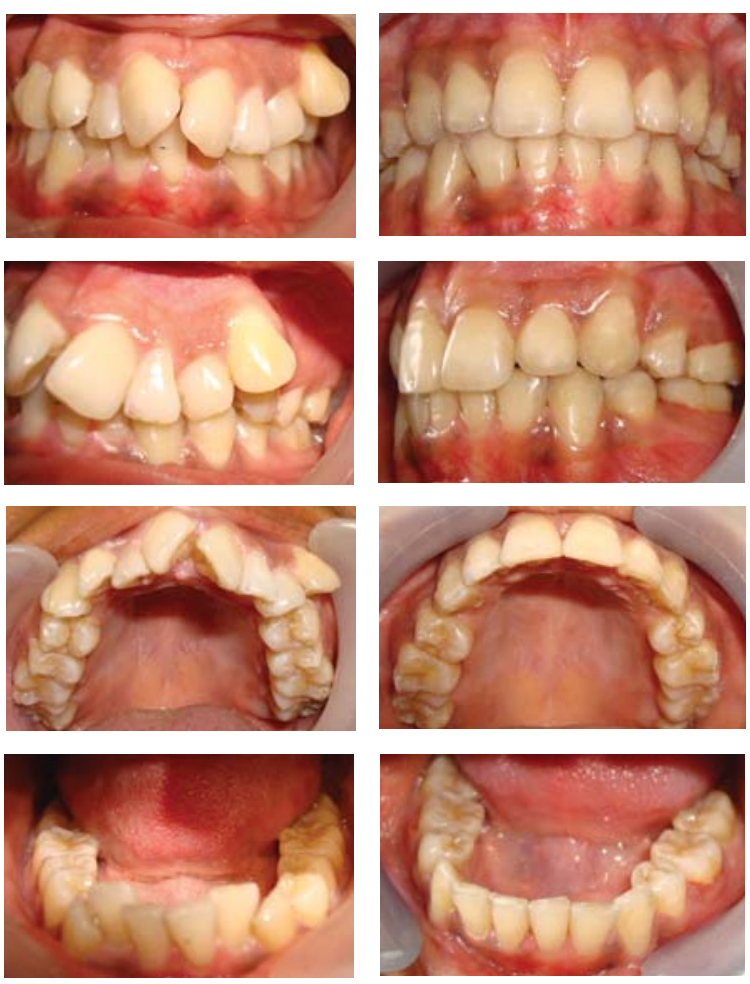

Fig. 9 Pre-post treatment extra oral photographs
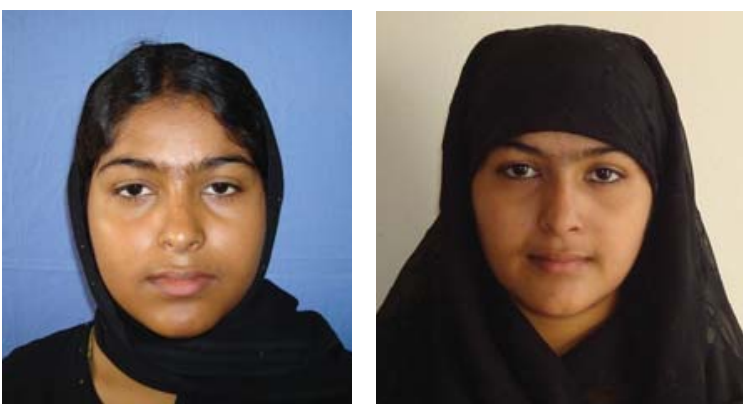


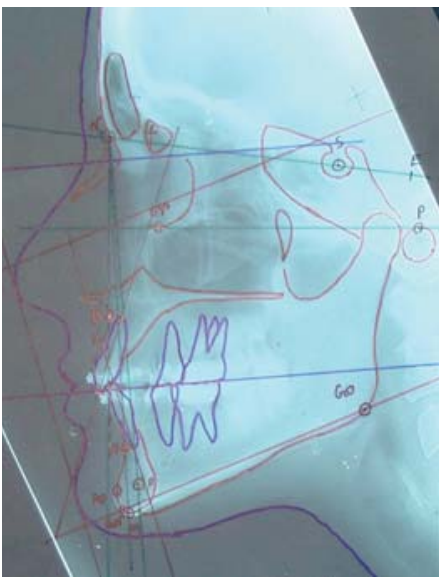

Fig 10: Post-treatment cephalometric tracing. Lower lip appears to be behind $\mathrm{E}$ line hence is normal in position

Table 2

\begin{tabular}{lcc}
\hline Parameter & $\begin{array}{r}\text { Pre treatment } \\
\text { measurements }\end{array}$ & $\begin{array}{c}\text { Post treatment } \\
\text { Measurement }\end{array}$ \\
\hline SNA (angle) & $84^{\circ}$ & $84^{\circ}$ \\
SNB (angle) & $81^{\circ}$ & $81^{\circ}$ \\
ANB (angle) & $3^{\circ}$ & $3^{\circ}$ \\
SND (angle) & $79.5^{\circ}$ & $79^{\circ}$ \\
Upper 1 to NA (mm) & $11.0 \mathrm{~mm}$ & $5.0 \mathrm{~mm}$ \\
Upper 1 to NA (angle) & $33^{\circ}$ & $16.5^{\circ}$ \\
Lower 1 to NB (mm) & $5.0 \mathrm{~mm}$ & $4.0 \mathrm{~mm}$ \\
Lower 1 to NB (angle) & $28^{\circ}$ & $15^{\circ}$ \\
Inter incisal angle & $117^{\circ}$ & $145^{\circ}$ \\
OP to SN (angle) & $14^{\circ}$ & $12^{\circ}$ \\
Go Gn to SN (angle) & $29^{\circ}$ & $29.5^{\circ}$ \\
SE (mm) & $21.5 \mathrm{~mm}$ & $23 \mathrm{~mm}$ \\
SL(mm) & $54.5 \mathrm{~mm}$ & $59 \mathrm{~mm}$ \\
\hline
\end{tabular}

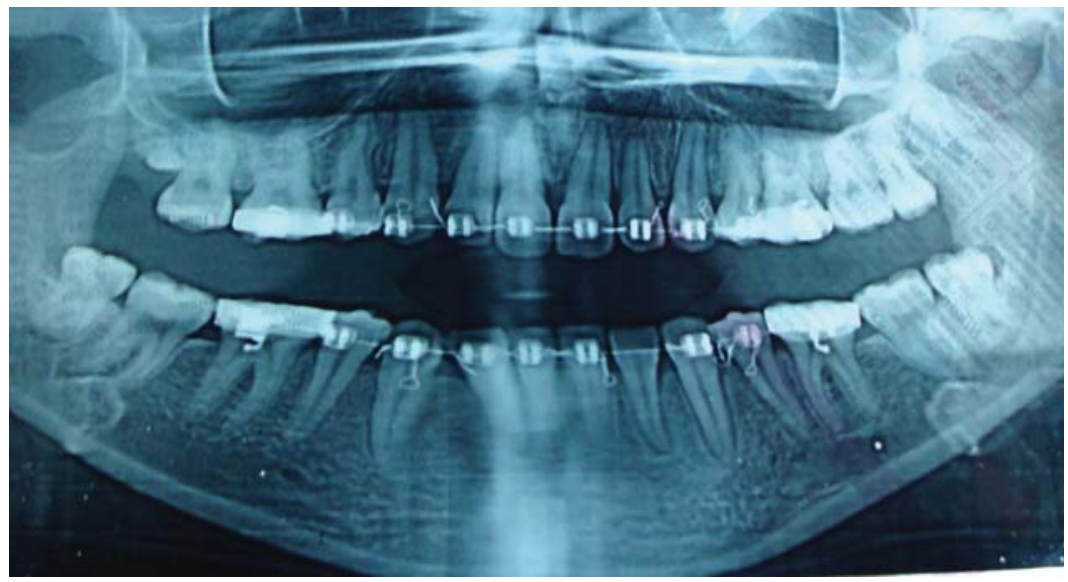

Fig 11: Post-treatment OPG

\section{CONCLUSION}

This case report has demonstrated with careful planning of extraction and orthodontic mechanics to deliver light, controlled force, condition of severe crowding can be corrected with good results.

\section{REFERENCES}

1. Boboc Gh: Anomaliile dentomaxilare. (Dentomaxillary anomalies) - in Romanian. Ed. Medicalã, Bucureti, 1971; pp 65-82.

2. Bishara SE: Textbook of Orthodontics, W.B. Saunders Company, 2001: pp 168-173, 442-444.

3. Chung $\mathrm{CH}$, Vanarsdall RL, Cavalcanti EA, Baldinger JS, Lai CH: Comparison of microbial composition in the subgingival plaque of adult crowded versus non-crowded dental regions. Int J Adult Orthodon Orthognath Surg., 2000; 15(4): 321-330.

4. Tanida K, Nagahara K, Iwata T: Orthodontic treatment of adults with periodontal disease. Aichi Gakuin Daigaku Shigakkai Shi., 1990; 28: 525-534. OHDMBSC - Vol. IV - No. 2 - June, 200527

5. Artun J, Osterberg SK: Periodontal status of secondary crowded mandibular incisors. Long-term results after orthodontic treatment. J Clin Periodontol, 1987; 14(5): 261-266.

\section{Correspondence-}

Dr. Hasan Md. Rizvi BDS, FCPS

Dental Surgeon,

Dept of Orthodontics

Dhaka Dental College and Hospital

Mobile : +88 01710918222 